\title{
SOME COMMENTS ON THE SIMPLE QUEUE
}

\author{
N. RAVICHANDRAN, ${ }^{*}$ Indian Insitute of Management, Ahmedabad
}

A. GRAVEY, ${ }^{* *}$ Centre National d'Études des Télécommunications

\begin{abstract}
This communication provides an explicit expression for the joint probability of exactly $i$ arrivals and $j$ service completions in a duration of length $t$ for the simple queue. The impossibility of a similar expression for the queue-length distribution is explained.

MARKOVIAN QUEUES: BUSY PERIOD: ARRIVAL PROCESS: DEPARTURE PROCESS: QUEUE-LENGTH DISTRIBUTION
\end{abstract}

\section{Introduction}

In a recent contribution Pegden and Rosenshine [3] have obtained an explicit expression for the joint probability of $i$ arrivals and $j$ service completions in $(0, t)$ for the simple queue. They also expressed concern about the possibility of a similar expression for the queue-length distribution. In this communication we provide an explanation for the impossibility of a closed-form solution for the queue-length distribution. Finally, an alternative approach for the joint probability mentioned is discussed.

\section{Some comments}

Consider the classical Markovian queue with parameters $\lambda$ and $\mu$. Let $A(t)(S(t))$ represent the number of arrivals (service completions) in $(0, t)$. Define $P_{i j}(t)$ as the joint probability that $A(t)=i$ and $S(t)=j$ with the condition that the number in the system initially is 0 . An explicit expression for $P_{i j}(t)$ is reported in [1]. We now provide a reason why such an expression is possible.

The stochastic process $\{A(t), S(t), t \geqq 0\}$ has the following properties:

1. For every $t, S(t) \leqq A(t)$.

2. The process $\{S(t), A(t)\}$ is a two-dimensional growth process.

3. The process stays in the state $(i, j)$ or moves to $(i+1, j)$ or $(i, j+1)$ after an exponential duration.

4. Transitions to states $(i, k), k>i$ and $(i, k), k<j$ from state $(i, j)$ are not possible. Hence, the event $\{A(t)=i$ and $S(t)=j\}$ is realized by a set of mutually exclusive and collectively exhaustive elementary events each of which corresponds to a path joining the origin to the point $(i, j)$ in a two-dimensional lattice, with the requirement that the number of horizontal steps is always as large as the number of vertical steps, and the step size in either direction is of unit length with no back tracking. The number $N$ of

Received 6 December 1983; revision received 14 August 1984.

* Postal address: Indian Institute of Management, Vastrapur, Ahmedabad-380 015, India.

** Postal address: CNET/EVP, BP 40, 22301 Lannion Cedex, France. 
such non-overlapping paths is obtained by using the reflection principle [2] as:

$$
N=\left(\begin{array}{c}
i+j-1 \\
i-1
\end{array}\right)-\left(\begin{array}{c}
i+j-1 \\
i+1
\end{array}\right)
$$

which is a finite number. Hence by writing down the path probabilities an explicit expression involving only finite sums results for $P_{i j}(t)$.

Now, it is possible to argue the reasons for the impossibility of a finite expression for the queue-length distribution. As is evident from [1], the $P_{i j}(t)$ functions are not useful.

If we follow the sample paths of the process $\{X(t), t \geqq 0\}$ representing the number in the system at any time $t$, then $X(t)$ is a simple birth and death process. However, before reaching the state $j$ the process may visit any state $k, k<j$ a non-finite number of times and each such realization will correspond to an elementary event, contributing to the event that the queue length is $j$. This is the main reason why a finite expression is not possible for the queue-length distribution. However, as explained earlier in the case of $P_{i j}(t)$ functions, such cycling is not possible and hence we obtain a finite closed-form expression for them.

\section{Another approach}

In this section an alternative approach for the joint probabilities $P_{i j}(t)$ is provided.

Let $\beta_{i j}(t)$ represent the probability that the number of arrivals (including the one at the origin) is $i$ and the number served equals $j$ with the provision that the server is continuously busy in $(0, t)$. To obtain an expression for $\beta_{i j}(t)$ observe that (a) The server is required to be busy in $(0, t)$ and (b) the number served should be equal to $j$. Further, during each service completion there will be a random number of arrivals and during the last uncompleted service time the number of arrivals is equal to the difference between $j$ and the accumulated arrivals during the previous service completions.

Hence,

$$
\begin{aligned}
\beta_{i j} & =0 \quad j>i \\
& =\lambda^{i-1} \mu^{j} \exp (-(\lambda+\mu) t) \frac{t^{i+j-1}}{(i+j-1) !}\left(\begin{array}{c}
i+j \\
i
\end{array}\right) \frac{i-j}{i+j} .
\end{aligned}
$$

Next, define $\pi(k, t) d t$ as the probability that a busy period ends in $(t, t+d t)$ with $k(\geqq 1)$ service completions given the initial condition that a busy period begins at $t=0$.

Using the $\beta_{i j}(t)$ and $\pi(k, t)$ functions with the observation that the server is busy in $(0, t)$ or not we write the following expression for $P_{i j}(t)$ :

$$
P_{i j}(t)=\left[\lambda e^{-\lambda t} * \sum_{k=1}^{i} \pi(k, t)\right] *\left[\lambda e^{-\lambda t} * \beta_{i-k j-k}(t)\right]+\lambda e^{-\lambda t} * \beta_{i j}(t),
$$

where $*$ denotes convolution.

It remains to evaluate the functions $\pi(k, t)$, which are determined by the following recurrence relation:

$$
\begin{gathered}
\pi(1, t)=\mu \exp (-(\lambda+\mu) t) \\
\pi(k, t)=b_{k}(t)+\sum_{j=1}^{k-1} \pi(j, t) * \lambda e^{-\lambda t} * b_{k-j}(t),
\end{gathered}
$$

where $b_{k}(t) d t$ is the probability that the busy period initiated at time $t=0$ is of length $t$ and the number served is $k$. An explicit expression for $b_{k}(t)$ is available in the literature even for the more general case of arbitrary service time durations (for example, see 
Cooper [1], p. 177) and is reproduced below:

$$
b_{j}(t)=\exp (-(\lambda+\mu) t) \mu \frac{(\lambda t)^{i-1}}{j !} \frac{\left(\mu_{t}\right)^{j-1}}{(j-1) !} .
$$

By using relations (3)-(6) an explicit expression for $P_{i j}(t)$ results.

It is worthwhile to observe that the entire set of relations is true even for the $M / G / 1$ queue by appropriate modifications in Equations (1) and (6).

\section{Acknowledgement}

N. Ravichandran acknowledges the financial support by CNET, Lannion during the preparation of this work.

\section{References}

[1] CoOPER, R. B. (1972) Introduction to Queuing Theory. Macmillan, London.

[2] Feller, W. (1968) An Introduction to Probability Theory and its Applications. Wiley, New York.

[3] Pegden, C. D. And Rosenshine, M. (1982) Some new results for the $M / M / 1$ queue. Management Sci. 28, 821-828. 